ESJ Social Sciences

\title{
Evaluación de la Calidad Pedagógica Enfocada en la Usabilidad de un Aplicativo Móvil Educativo en Estudiantes de Educación Superior Tecnológica de Ecuador
}

\author{
Darío Xavier Romero Santistevan \\ Ingeniero en Electrónica y Telecomunicaciones, Maestria en Ingeniería \\ Biomédica, Docente del Instituto Superior Tecnológico Juan Bautista \\ Aguirre \\ Silvania Gabriela Herrera Martínez \\ Licenciada en ciencias de la educación mención Lengua Inglesa y \\ Linguística, Directora del Departamentos de Idiomas del ISTSB, Instituto \\ Superior Tecnológico Simón Bolívar \\ Ronald Darwin Vélez Zambrano
}

Ingeniero en electrónica y telecomunicaciones, Magister en Conectividad y Redes de Telecomunicaciones, Docente del Instituto Superior Tecnológico

Juan Bautista Aguirre

Jhon Hennry Mendoza Cedeño

Magíster en gerencia educativa, Lcdo. En psicología y orientación vocacional, Unidad Educativa Fiscal Juan León Mera

Cesar Israel Mendoza Moyón

Licenciado en Ciencias Navales, Magister en Administración de Empresas, Instituto Oceanográfico y Antártico de la Armada, Armada del Ecuador

Doi:10.19044/esj.2021.v17n35p70

Submitted: 03 August 2021

Accepted: 29 September 2021

Published: 31 October 2021
Copyright 2021 Author(s)

Under Creative Commons BY-NC-ND

4.0 OPEN ACCESS

Cite As:

Romero Santistevan D.X., Herrera Martínez S.G., Vélez Zambrano R.D., Mendoza Cedeño J.H. \& Mendoza Moyón C.I. (2021). Evaluación de la Calidad Pedagógica Enfocada en la Usabilidad de un Aplicativo Móvil Educativo en Estudiantes de Educación Superior Tecnológica de Ecuador. European Scientific Journal, ESJ, 17 (35), 70.

https://doi.org/10.19044/esj.2021.v17n35p70

\section{Resumen}

El presente estudio se enfoca en la necesidad que tienen los estudiantes como la brecha digital que les impide desarrollar un uso efectivo de herramientas tecnológicas y que las instituciones de educación superior en Ecuador deben de implementar dichas tecnologías del aprendizaje como 
herramientas didácticas para el fortalecimiento del conocimiento y la simulación de situaciones con apego a la realidad. Por tanto, el objetivo de esta investigación fue evaluar la usabilidad que tiene una aplicación móvil como herramienta para el aprendizaje por medio del análisis de descriptores: a. Pertinencia, b. Facilidad de uso, c. Personalización, d. Retroalimentación, e. Autenticidad, f. Habilidades del pensamiento, g. Trabajo colaborativo y h. Motivación, en función al desempeño pedagógico de un nuevo entorno como lo son las aplicaciones móviles. En consecuencia, se detalló una metodología con enfoque cualitativo por medio de un diseño no experimental y método inductivo basado en la descripción de las experiencias generadas por estudiantes con el aplicativo móvil de la institución. Como instrumento se seleccionó la encuesta estructurada por una escala de Likert valorada de (1-5) partiendo de "imperceptible" hasta "fuerte" en función a la aceptación de los ocho descriptores por parte de los encuestados. Por lo tanto, se planteó como población de estudio 1408 estudiantes y por medio de un muestreo probabilístico se calculó una muestra de 302. Así mismo, varios resultados pusieron a prueba las ventadas como también desventajas y dificultades que generó el aplicativo en el proceso de aprendizaje donde el 29,5\% de los estudiantes determina una motivación fuerte en el uso del aplicativo móvil, sin embargo durante el ejercicio de las clases el 59,3\% de los estudiantes han percibido frustración debido a la conectividad o al colapso del aplicativo.

Palabras claves: Usabilidad, Pertinencia, Personalización, Retroalimentación, Habilidades de pensamiento 


\title{
Assessment of Pedagogical Quality Focused on the Usability of an Educational Mobile Application in Technological Higher Education Students
}

\author{
Darío Xavier Romero Santistevan \\ Ingeniero en Electrónica y Telecomunicaciones, Maestria en Ingeniería \\ Biomédica, Docente del Instituto Superior Tecnológico Juan Bautista \\ Aguirre \\ Silvania Gabriela Herrera Martínez \\ Licenciada en ciencias de la educación mención Lengua Inglesa y \\ Linguística, Directora del Departamentos de Idiomas del ISTSB, Instituto \\ Superior Tecnológico Simón Bolívar \\ Ronald Darwin Vélez Zambrano
}

Ingeniero en electrónica y telecomunicaciones, Magister en Conectividad y Redes de Telecomunicaciones, Docente del Instituto Superior Tecnológico Juan Bautista Aguirre

\section{Jhon Hennry Mendoza Cedeño}

Magíster en gerencia educativa, Lcdo. En psicología y orientación vocacional, Unidad Educativa Fiscal Juan León Mera

\section{Cesar Israel Mendoza Moyón}

Licenciado en Ciencias Navales, Magister en Administración de Empresas, Instituto Oceanográfico y Antártico de la Armada, Armada del Ecuador

\begin{abstract}
This study focuses on the need that students have, such as the digital divide that prevents them from developing an effective use of technological tools and that higher education institutions in Ecuador must implement these learning technologies as didactic tools for the strengthening of knowledge. and the simulation of situations with attachment to reality. Therefore, the objective of this research was to evaluate the usability of a mobile application as a tool for learning through the analysis of descriptors: a. Relevance, b. Ease of use, c. Personalization, d. Feedback, e. Authenticity, f. Thinking skills, g. Collaborative work and $\mathrm{h}$. Motivation, based on the pedagogical performance of a new environment such as mobile applications. Consequently, a methodology with a qualitative approach was detailed through a nonexperimental design and inductive method based on the description of the experiences generated by students with the institution's mobile application. As an instrument, the structured survey was selected by a Likert scale valued from (1-5) starting from "imperceptible" to "strong" based on the acceptance of the eight descriptors by the respondents. Therefore, 1408 students were proposed
\end{abstract}


as the study population and a sample of 302 was calculated through probabilistic sampling. Likewise, several results tested the advantages as well as disadvantages and difficulties that the application generated in the learning process where $29.5 \%$ of the students determine a strong motivation in the use of the mobile application, however during the exercise of the classes $59.3 \%$ of the students have perceived frustration due to the connectivity or the collapse of the application.

Keywords: Usability, Relevance, Personalization, Feedback, Thinking skills

\section{Introduction}

En la actualidad la sociedad guarda mucha relación y dependencia hacia el uso de dispositivos tecnológicos, por ejemplo, teléfonos celulares, entre otros. Además, los individuos son miembros inmediatos como especialistas independientes, ya que realizan un uso máximo de datos avanzados a través de estos medios sin tener la oportunidad de realizar o reclasificar las TIC, según lo indiquen sus inclinaciones (no se apropia de la innovación). Las condiciones universitarias no se liberan del efecto de estas progresiones en nuestro período informatizado; los educadores y los estudiantes pueden beneficiarse de la consolidación de reliquias avanzadas que funcionan con las condiciones de aprendizaje y las mejoran (González D. , 2017).

La medida de aprendizaje educadora intervenida por las TIC hace concebible ajustarse a los requerimientos y atributos de los clientes en su preparación e inclinaciones. No es extraño discutir esta temporada de progreso, lograda por impulsos en los avances de datos y correspondencia, como el comienzo de otro período, al que se alude regularmente como la sociedad de datos. Con el libre ingreso a la innovación, es factible planificar y establecer condiciones virtuales que se ajusten y se alteren a las necesidades instructivas; que trabajen con el esfuerzo coordinado de los pensamientos y aumenten el tiempo disponible para el giro de los acontecimientos, la utilización y determinación de la sustancia, según sus requerimientos (Castro \& Guzmán, 2007).

Las condiciones a nivel educativas han sufrido una transformación total debido a la pandemia provocada por el virus del covid-19 sin embargo, la tecnología ha surgido como un mecanismo que puede aportar en el desarrollo de los procesos de aprendizajes. A pesar de esto, la innovación por sí sola no cambia el modelo instructivo, las prácticas de instrucción o los estándares actuales; no es apto para tomar atención a los problemas y dificultades que inundan las situaciones universitarias. Fundamentos instructivos utilizan el aprendizaje de Internet, como él hace referencia, para 
seguir haciendo ensayos de aprendizaje similares sin atender a nuevas necesidades (Pujals, 2018).

Se utilizan nuevos avances para seguir replicando antiguos orígenes instructivos. No hemos recordado que el tema del aprendizaje es un ser innovador insuperable. Se debe poner énfasis en la educación, en los procedimientos de instrucción de los instructores, en la correspondencia y difusión de los marcos de aprendizaje (fomentando las medidas de desarrollo sostenidas por las TIC) y no en centrar los esfuerzos en la accesibilidad mecánica y el potencial (Rincón, 2016).

La adquisición de innovación y el uso básico de la innovación no cambia la escena educativa. Es importante capacitar a los docentes, como cuestión de primera en la importancia en el procesos de aprendizaje y enseñanza actual, como también generar en los estudiantes las habilidades, destrezas y dominio esenciales para ayudarlos a descubrir enfoques simular los beneficios que genera la presencialidad. Dichas características que les permitirán que los estudiantes y la escuela afronten las dificultades y necesidades en esta nueva era. Por tanto, las TIC se suman al cambio necesario donde docenes y estudiantes requieren nuevas habilidades y cambios en las metas, para que puedan contribuir a su autoridad y logro respectivamente (Rodríguez, 2018).

Por otra parte, algunas organizaciones se comprometieron con la evaluación y ejecución de la gamificación en los productos como también en la administraciones de las mismas, con el objetivo específico de expandir el compromiso y la devoción con respecto a sus clientes y trabajadores; Algunas instancias las marcas que han utilizado adicionalmente la gamificación en sus aplicaciones versátiles son: programación AppleWatch, programación Nike +, Waze Navigator, Booking supervisor de reservas Booking y Uber Transportation System; específicamente estas aplicaciones portátiles han descubierto cómo hacer y mantener otra propensión a sus compradores aumentando el valor de los artículos y las administraciones que ofrecen utilizando la estrategia de gamificación. El método de Gamificación no es restrictivo para el área comercial o de exhibición, este procedimiento también es apropiado en diferentes regiones. En el campo instructivo, la Gamificación tiene una rama comprometida con la investigación de la utilización de partes del juego dentro del ciclo de aprendizaje, esta rama se clasifica como "Aprendizaje basado en juegos", esta rama permite al suplente probar su mantenimiento e información, a través de métodos más potentes y ejercicios de prueba que los que se utilizan actualmente en el entrenamiento convencional anunciado (Ortiz, Colón, \& AgredaI, 2018).

La UNESCO alude a los Recursos Educativos Abiertos (REA) como la disposición abierta de los activos instructivos intercedidos por las TIC para el asesoramiento, uso y transformación por parte de un área local de clientes 
con fines no comerciales. Los REA son componentes o materiales en diseño computarizado que se apropian de manera complementaria para su uso en la medida de aprendizaje de la instrucción, tanto por parte de instructores y suplentes como por parte de científicos. La dispersión de estos activos se realiza mediante licencias abiertas o Creative Commons para trabajar con su utilización, variación, inducción y reordenamiento público (UNESCO, 2019).

Estos activos potencian su utilización, actualización y personalización para fines de preparación, de los cuales es factible distinguir los aspectos más destacados y los objetivos de la didáctica del instructor para la disposición y desarrollo de la información por parte del suplente. bajo licencias abiertas, el cliente (suplente) puede crear sus propias variantes y recomendaciones de activos y sustancia. En este examen se consideran tres ángulos fundamentales para describir un REA: (1) instructivo, donde se consideran enfoques que permiten el aprendizaje dinámico a través de la gamificación y la correlación. (2) Digital, que permite la codificación de datos en un doble lenguaje para la creación y distribución del activo en un escenario virtual. (3) El abierto, que trabaja con la accesibilidad de activos en una posición de libre (Gallegos, 2015).

Un clima de aprendizaje virtual se retrata como un espacio de datos destinado a una interacción instructiva, donde los animadores transmiten según las reglas académicas que dirigen la mejora de puntos específicos con una razón de aprendizaje. Para su origen y ejecución, se separan un montón de segmentos, medidas y puntos de vista de marcas. La tabla adjunta resume una parte de estos componentes que se encuentran en la auditoría escrita (Naranjo, 2019).

Tabla 1 : Conjunto de componentes y dimensiones de un ambiente virtual

\begin{tabular}{|c|c|c|c|}
\hline Clasificación & Propuesta - Categoría & Propósito & Fuente \\
\hline Dimensiones & $\begin{array}{l}\text { a) Orientación epistemológica } \\
\text { general, b) modelos psicológicos } \\
\text { y educativos de referencia; c) } \\
\text { Dominio de conocimiento; d) rol } \\
\text { del profesor; e) rol de aprendiz; y } \\
\text { d) nivel de autenticidad de las } \\
\text { actividades diseñadas. }\end{array}$ & $\begin{array}{c}\text { Analizar } \\
\text { ambientes de } \\
\text { aprendizaje }\end{array}$ & $\begin{array}{c}(\text { Coll \& } \\
\text { Monereo, 2008) }\end{array}$ \\
\hline Elementos & $\begin{array}{l}\text { a) Organizaciones, comunidades; } \\
\text { b) actividades; y c) prácticas. }\end{array}$ & $\begin{array}{l}\text { Caracterizar un } \\
\text { ambiente virtual }\end{array}$ & $\begin{array}{c}\text { (Valencia, } \\
\text { huertas, \& } \\
\text { baracaldo, 2014) }\end{array}$ \\
\hline Etapas & $\begin{array}{l}\text { a) Diagrama visual del contenido; } \\
\text { b) corrección; c) análisis de } \\
\text { interoperabilidad entre sistemas y } \\
\text { plataformas; d) cumplimiento de } \\
\text { estándares; e) generación de } \\
\text { contenido en otros idiomas; f) } \\
\text { testeo y control de calidad; y g) } \\
\text { arquitectura de la información. }\end{array}$ & $\begin{array}{l}\text { Definir las etapas } \\
\text { del proceso de } \\
\text { producción de } \\
\text { contenido }\end{array}$ & $\begin{array}{c}\text { (Pérez- } \\
\text { berenguer \& } \\
\text { garcía-Molina, } \\
\text { 2016) }\end{array}$ \\
\hline
\end{tabular}




\begin{tabular}{|c|c|c|c|}
\hline Espacios & $\begin{array}{l}\text { a) Interactividad tecnológica } \\
\text { potencial; b) Interactividad } \\
\text { pedagógico potencial; c) } \\
\text { Interactividad tecnológica real; y } \\
\text { d) Interactividad pedagógica real. }\end{array}$ & $\begin{array}{l}\text { Analizar } \\
\text { referentes } \\
\text { potenciales y } \\
\text { reales del } \\
\text { ambiente } \\
\text { educativo. }\end{array}$ & $\begin{array}{l}\text { (Mauri, onrubia, } \\
\text { Coll, \& } \\
\text { Colomina, } \\
\text { 2016) }\end{array}$ \\
\hline $\begin{array}{c}\text { Componentes } \\
\text { operativos }\end{array}$ & $\begin{array}{l}\text { a) Comunicación; b) } \\
\text { Productividad; c) para involucrar } \\
\text { al estudiante; d) entrega de cursos } \\
\text { y evaluación del aprendizaje; e) } \\
\text { colaboración en línea; f) } \\
\text { Desarrollo de cursos. }\end{array}$ & $\begin{array}{l}\text { Identificar las } \\
\text { cualidades de } \\
\text { cada entorno }\end{array}$ & (Landon, 1989) \\
\hline
\end{tabular}

Nota: Información tomada a partir de las fuentes citas en la misma tabla.

Además, las nuevas innovaciones son instrumentos que pueden sumarse a la obtención de información, surgiendo el e-picking como un mecanismo que permite al estudiante seguir aprendiendo fuera del aula, a pesar de que el educador es todavía fundamental para establecer el orden dentro del aula; por otra parte, se ha distinguido que el m-learning puede ser un factor fundamental para asegurar la cooperación de los adultos jóvenes para familiarizarse con las necesidades que las técnicas habituales no han logrado (Aguiar, Velázquez, \& Aguiar, 2019).

A pesar de esto, existen algunos obstáculos para la unión de la innovación instructiva en la educación avanzada, que incluyen: la base, el esfuerzo del educador, el cumplimiento con su utilización y la priorización de estos dispositivos en ejercicios distintos al aprendizaje. Del mismo modo, aunque el m-learning se está extendiendo rápidamente en numerosos lugares del mundo, la investigación sobre las variables que impulsan su recepción es escasa (Herrera M. , 2018).

De tal manera, que el docente debe de liderar los procesos de enseñanza y aprendizaje por medio del uso de los dispositivos tecnológicos para que él mismo establezca los límites y n permita que el estudiante abuse con el control de los dispositivos (que son amplios en el campo del e-learning), por tal razón los educadores que eventualmente son el canal a través del cual se garantiza la cita de información; significativamente más cuando los nuevos avances han representado una progresión de dificultades para los marcos instructivos, donde la obstrucción de numerosos eventos a los cambios de la visión del mundo está más relacionada con los entrenadores que con los estudiantes (Mujica, 2020).

\section{Ventajas del aprendizaje electrónico}

Este apartado presenta los beneficios que ofrece la preparación en internet o la educación a distancia. En este sentido, los establecimientos de educación superior deben evaluar las estructuras que ha tomado la formación 
a distancia, ya que las intranets y las redes de amplia región se han creado y resultan más ineludibles, dado que las estrategias de e-learning son tan viables para la instrucción, o considerablemente más que los salones de clases convencionales. De ahora en adelante, pueden otorgar libertades de aprendizaje a personas que en cualquier caso no se acercarían a la nueva información; Además, las combinaciones de sala de estudio y e-learning podrían ser una metodología superior en establecimientos instructivos. En este caso, llaman la atención sobre el acompañamiento como ventajas del elearning: (Carneiro, Toscano, \& Díaz, 2021).

- Ahorro. El e-learning infiere una enorme disminución de gastos y costes cuando se contrasta con el aprendizaje vis-à-vis, ya que con el aprendizaje por internet se logran considerables fondos de reserva en cuanto a transporte, materiales de exposición, entre otros.

- Rapidez y destreza. Depende del gigantesco beneficio de ser una interacción de aprendizaje donde prevalece la prontitud en cuanto a conseguir los datos importantes para un aprendizaje. Los datos son accesibles en el momento, de forma ágil y realmente disponible.

- Acceso justo a tiempo. La preparación a través del e-learning se realiza en el momento en que se requiere. En este sentido, el cliente controla fundamentalmente la hora de su preparación basada en la web.

- Acceso sólo para mí. Además de tener la opción de realizarlo cuando se necesita, se trabaja con un tipo de educación sustancialmente más individualizada y personalizada, tal y como indican los atributos académicos e innovadores.

- Material electrónico. Sustituye el diseño en papel por una configuración electrónica. La mayoría de las veces se trata de material multimedia variado como, por ejemplo, ejercicios de instrucción en vídeo o introducciones, entre otros.

- Barrera espacial. Con el e-learning, los obstáculos espaciales desaparecen, ya que es posible realizar cualquier tipo de preparación en Internet sin tener en cuenta la zona geográfica del cliente. Es lo que se conoce como deslocalización espacial.

- Actualización. En el e-learning los contenidos están en la red, es decir, tienen la ventaja de estar facilitados en espacios donde pueden ser revisados y actualizados continuamente. En este sentido, el beneficio no es ciertamente un refresco unidireccional, sino que tiende a ser terminado por los dos instructores y los estudiantes

- Multigadget. El e-learning permite el aprendizaje de una enseñanza desde un amplio abanico de gadgets. Con el e-learning es posible acceder a cualquier preparación desde un teléfono móvil, una tableta o un PC 
- Conciliación. Al ser un tipo de educación deslocalizada, el e-learning permite algo crucial en la sociedad actual: la conciliación entre el día a día y la vida laboral. El e-learning hace posible que el aprendizaje y el día a día sean viables, fundamentalmente porque permite el plan adaptable al que se ha hecho referencia anteriormente

- Tutorización. Es un componente principal del e-learning. El hecho de que los alumnos no puedan tener contacto directo con el profesor que imparte el curso no impide que se gestione el avance del alumno a través de la tutoría. Hay algunas formas en las que los alumnos pueden tener contacto líquido con el educador/facilitador a través del correo electrónico o la videoconferencia, entre otros.

- Aprendizaje colaborativo. Aunque en el e-learning el aprendizaje es principalmente individual, esto no impide que determinados tipos de instrucción se centren en lo que se conoce como aprendizaje comunitario.

- Se trata de un aprendizaje virtual, pero en el que, a través de una etapa concreta, varias personas pueden realizar ejercicios, debates y conceptualizaciones para compartir pensamientos. Una ilustración razonable de un escenario comunitario sería Google Hangouts.

- Plataforma virtual. A pesar de ser una asociación de Internet, al igual que un aparato electrónico, en e-learning no es inesperado utilizar algún tipo de escenario donde se concentran todos los datos identificados con la instrucción. En la formación, muy posiblemente el escenario más combinado es Moodle

- Gestión de la información. La administración de los datos a través del e-taking no es, sin duda, la misma que la de la preparación en casa. En el e-learning el alumno parte de un gran volumen de datos al que tiene libre acceso.

- Titulación. Un número cada vez mayor de lecciones ayudadas a través del e-learning tienen una capacidad de autorización muy parecida a la de cualquier preparación en casa. Este punto de vista ha impulsado sin duda a numerosas personas a seleccionar este tipo de aprendizaje electrónico.

Pensando en la sustancia a la que se hizo referencia recientemente, es importante alentar a las organizaciones de educación avanzar en la adquisición de nuevas plataformas web y el e-learning, ya que ofrecen la forma de ayudar a la preparación y capacitación que se suma al intercambio de información, ya que estas nuevas modalidades en la instrucción pueden crear una integración entre el estudiante y docente desde un enfoque colaborativo que convierte a 
este tipo de actividades en un puente de mejora a nivel sociedad (Oeganización Mundial del Comercio, 2018).

\section{Desventajas del aprendizaje electrónico}

Con relación a las desventajas Leiva (2016) describe que el aprendizaje electrónico móvil está basado en redes inalámbricas para el acceso al contenido educativo de forma efectiva proporcionando el intercambio de ideas para el proceso de adquirir nuevos conocimientos, sin embargo contar con conexiones a internet que no abastecen las necesidades educativas limita a los estudiantes y docentes la implementación de aquellas herramientas a favor de la educación o formación virtual. En cuanto a redes y tecnología incide además que dispositivo móvil utiliza el usuario ya que cada recurso cuenta con características diferentes el cual cambia el rendimiento, como son la duración de carga eléctrica, seguridad de contenidos, conectividad inalámbrica, sistema operativo, visibilidad de contenido puesto que generalmente cuando se refiere a un smartphone es de dimensiones reducidas y esto puede acarrear cierta dificultad al momento de proyectar información o no es compatible para el tipo de tecnología (Mejía, 2020).

Los factores o recursos identificados anteriormente pueden llegar a limitar las posibilidades del uso de dispositivos móviles como los smartphones, por parte de Ávila (2019) describe como una desventaja el déficit de desarrollo de las destrezas receptoras de los conocimientos curriculares, es decir al momento de tomar la decisión de continuar con el uso de tecnología para conocimientos significativos a nivel profesional como lo son en la educación superior es imprescindible contar con habilidades tanto comunicativas como digitales para todos los partícipes que además inciden en el proceso de aprendizaje. Con relación a los docentes un requisito indispensable en su formación continua es poseer destrezas digitales que facilite alcanzar las metas y resultados significativos establecido en las planificaciones curriculares, además el rol de los docentes es fundamental para el reconocimiento como también la identificación de inconvenientes para su posterior aclaramiento debido a que los estudiantes podrían no contar con un nivel de habilidades relacionadas con la asignatura.

Así mismo, los smartphones como dispositivos tecnológicos en el uso dentro del ámbito educativo no fueron tan aceptado por parte de las instituciones de educación superior, así lo demuestra Mejía (2020) puesto que poseen una gran variedad de aplicaciones que actúan como herramientas dependiendo de calidad y costos. Sin embargo, la implantación de los teléfonos inteligentes tendría repercusiones si en su debido caso no se da un seguimiento al proceso de aprendizaje ya que en lugar de generar conocimiento llegan a ser distractores por lo tanto es preferible marcar parámetros sin la necesidad de prohibición. Por tal motivo, los smartphone 
según los expertos educativos deberían tomarse medidas regulatorias para evitar que la tecnología genere distracciones y desatienda las necesidades educativas, por ejemplo, permitir a los estudiantes el acceso a los dispositivos móviles cuando se requiera.

En base a lo anterior mencionado no cabe duda de que organizar y planificar un programa pedagógico adecuado encamina a resultados satisfactorios apegados a los objetivos educativos, sin embargo, existen ciertos criterios a tomar en cuenta que a futuro evitan estancar el aprendizaje mediante teléfonos celulares, por lo tanto, es necesario encontrar los instrumentos para maximizar su utilidad es decir buscar soluciones y evitar inconvenientes.

\section{Aprendizaje electrónico móvil}

Para abordar este punto, es importante explicar algunos términos. Así, el aprendizaje versátil, también llamado M-learning, alude a las condiciones de aprendizaje que dependen de la innovación portátil, apuntadas a mejorar y promover las medidas de instrucción y aprendizaje. En consecuencia, se puede ver muy bien que el aprendizaje polivalente se identifica con la escolarización fuera de la sala de estudios, es decir, la formación a distancia (Cruz, Aguilar, Colomo, Gabarda, \& Domínguez, 2018).

Desde otro punto de vista, Kinshuk (2009) advierte que se trata de un patrón que surge debido a la multiplicación de los marcos de correspondencia actuales. En lo que a él respecta, Quinn (2010) lo imagina como la conjunción entre el registro portátil y el e-learning, pero con apertura a los activos necesarios, así como un soporte de aprendizaje viable según Brazuelo Grund y Gallego Gil (2011), muestran que El M-Learning se presenta como una ayuda para ciclos instructivos polivalentes, que requieren una alta inteligencia en la mejora del aprendizaje, con conciliación de contenidos y universalidad en los ejercicios psicológicos (García \& Corona, 2020).

Actualmente, en circunstancias como la actual, se induce lo innato en la innovación intelectual, de tal manera que Cavaller (2016), remarca que ese es el camino al desarrollo; en consecuencia, los creadores lo imaginan como "La línea que aísla las consecuencias del examen mecanizado y la innovación psicológica es generalmente leve", ya que se conocen una gran cantidad de los avances ocultos del registro intelectual -como el aprendizaje profundo, en (IA) y las organizaciones neuronales, lo que lo convierte en una base para la recogida adaptable y compartida, convirtiéndose en un dispositivo importante para la dinámica.

Como indican las hipótesis planteadas, muy bien se puede ver que el aprendizaje electrónico versátil ofrece técnicas actuales de ayuda a la medida de aprendizaje instruccional mediante el uso de teléfonos móviles, por ejemplo, Tablet-PC, Mano derecha avanzada personal (PDA), MP3, PDA (Smartphone), celulares y computadoras, entre otros. En resumen, se suele 
advertir que el aprendizaje electrónico portátil se introduce como un componente constantemente conectado a cualquier dispositivo portátil que tenga algún tipo de disponibilidad remota, convirtiéndose en un aparato típico en las existencias de numerosos individuos, donde se está convirtiendo en una respuesta a los problemas que enfrenta el ámbito escolar (Cavaller, 2016).

En este sentido, los interlocutores en la medida de aprendizaje educativo establecen una ayuda ya que, en su directo, la orientación se puede completar en cualquier lugar, como en cualquier momento. Por lo tanto, la utilización de teléfonos celulares, al igual que los nuevos avances, se suman a la orientación formal y casual, ya que posiblemente pueden cambiar la ejecución y la preparación instructivas, de ahora en adelante el significado de este examen (Chiquinquirá \& Hernández, 2020).

\section{Ventajas y desventajas del aprendizaje electrónico móvil en la educación superior}

En la práctica educativa, las nuevas tecnologías han presentado diferentes dispositivos como también softwares que podrían facilitar el acceso a una educación facilitando en relación con el tiempo y dinero a los estudiantes. En primera instancia se desarrolló la educación a distancia en la cual se tomó como medio la tecnología sin embargo, esta representaba grandes retos en relación a calidad educativa llevando a otro nivel la tecnología aplicada a la educación pero para eso fue necesario que tanto estudiantes como docentes tengan los conocimientos para el manejo de dichos instrumentos creando así una brecha tecnológica para quienes podían acceder a las plataformas como también la adquisición de infraestructura y equipos importantes e indispensables para el desarrollo educativo en modalidades virtuales o diferentes a la presencial (Carneiro, Toscano, \& Díaz, 2021).

Dentro de este contexto, Hernández \& Morales (2010), señalan varias utilidades e inconvenientes en la aplicación del M-Learning. En este sentido, dentro de las bondades en la aplicación de la metodología indicada, se tiene:

- Aumento del tiempo útil y de la disponibilidad geográfica.

- Se puede ampliar la oportunidad de crear ejercicios educativos y de aprendizaje.

- Mayor autosuficiencia.

- Los estudiantes pueden modificar el teléfono celular según sus necesidades.

- Contenido ajustado al área real

- El celular puede mostrar los datos de lo más cercano a él respecto a la hora de la que necesitas obtener datos, intereses explícitos o hacia dónde te diriges.

- Necesidad de asociación. 
- El acceso inalámbrico se puede escalar de manera útil para varios clientes.

- Ingreso inmediato a información y toma nota.

- Los usuarios pueden acceder rápidamente a cierta información que deben transmitir en un breve período de tiempo, entre otros.

- Confirmación segura.

- Los dispositivos móviles pueden ser un instrumento útil para distinguir al cliente y algunas técnicas de pago adaptables.

- Alta personalización.

- Presenta un entorno virtual con un panorama abierto para que los estudiantes puedan indagar y descubrir nuevas aplicaciones en función al desarrollo del aprendizaje.

- Alto desarrollo de la innovación.

No obstante lo mencionado anteriormente, la forma en que el desarrolla la innovación trae consigo algunas barreras dentro de la adaptabilidad tecnológico desde la perspectiva de los estudiantes como también de los docentes:

- Brecha digital

- Analfabetismo digital

- Retroceso en el proceso de aprendizaje

- Alto costo en la adquisición de nuevos equipos tecnológicos (hardware y software).

- Inconvenientes con el acceso a internet.

Continúan resaltando Hernández \& Morales (2010), sobre algunos de los inconvenientes en uso del MLearning, dentro de los cuales se tiene:

- Resistencia al cambio por parte de ciertos suplentes o instructores.

- Dificultad para adaptarse a los teléfonos móviles.

- Falta de periféricos para el celular que limitan al cliente a modelos de aprendizaje esenciales.

- A excepción de las Tablet PC y las estaciones de trabajo, tienen problemas relacionados con la facilidad de uso debido a las pantallas pequeñas.

- La navegación suele estar restringida.

- Los costos de acceso a la red son altos.

- El trabajo colaborativo es menor.

- Necesidad de asociación remota que pueda verse influenciada por el área real del cliente. 
A la luz de los pensamientos planteados, los científicos muestran las ventajas de MLearning; desde entonces, se presentan como otra cosmovisión para el aprendizaje comunitario. En esta línea, las condiciones constructivistas tienen ventajas y también inconvenientes ya que la utilización de esta metodología permitirá al estudiante realizar los ejercicios a su propio ritmo, ya que cada estudiante realmente querrá percibir la importancia de asumir compromisos que le reportarán beneficios. En resumen, uno de los objetivos preestablecidos en la instrucción contemporánea se cumpliría, ya que, al enfocarse en un número crítico de estudiantes, reaccionaría con un interés insatisfecho, así como expandir y trabajar con los resultados potenciales de la educación para aquellos individuos que por diferentes razones no puede ir a las condiciones de entrenamiento adecuadas habituales (Herrera B. , 2012).

\section{M-Learning en la educación superior}

Durante bastante tiempo, el modelo instructivo que depende de la utilización de teléfonos móviles se ha basado en varios tipos de comprensión, que pueden cambiar dependiendo de las cualidades de cada uno de ellos. No obstante, no son selectos, y pueden integrarse y lograr ejemplos combinados que tome lo mejor de cada tipo para hacer uno más completo (Hernández \& Morales , 2010).

En lo que a él respecta, Hernández \& Morales (2010) destaca que la utilización del M-Learning en la sala de estudio trabaja con la comprensión de la información; Incorpora la vista y el sonido y se centra en el clima, incita al respeto por la variedad, así como favorece el aprendizaje centrado en el suplente y en la circunstancia concreta. Por otra parte, potencia la colaboración y la correspondencia, además de permitir el intercambio de información entre personas del ámbito local instructivo (suplentes e instructor), da la distribución inmediata de sustancia y comentarios, entre otros.

Asimismo, Hernández \& Morales (2010) destaca que el M-Learning es una situación virtual donde el desarrollo de la información comprende una interacción funcional ya que es a partir de la experiencia de suplentes y guías online que se genera una mejora única de tipo social, pero con importancia escolar. Por lo tanto, las técnicas de seguimiento o verificación deben evaluarse, al igual que para mantener el control de las aulas virtuales, la filosofía de M-Learning aborda un aparato fenomenal que proporciona registros que ayudan a medir la ejecución de los suplentes. En este sentido, con esta aplicación, la fundación instructiva intenta lograr una mejora constante. En este caso, el binomio escolar e innovador en este enfoque comprende una interacción funcional donde las partes que intervienen (establecimiento de educación avanzada, mentor y suplentes) asumen una parte dinámica e inteligente. 
En otro orden de cosas, Herrera (2012) advierte que el uso del MLearning es apremiante para el aprendizaje dependiente del pensamiento crítico, para la obtención de habilidades, en el trabajo de campo, en los exámenes exploratorios, entre otros. Desde otro punto de vista, Marqués (2013) sostiene que esta filosofía de aprendizaje versátil sostiene la evaluación evolutiva y sumativa, además de expandir la inspiración del suplente. En resumen, el aprendizaje electrónico versátil aborda una ventana innovadora para la metodología instructiva, que suma al desarrollo de la información, el objetivo de los problemas de aprendizaje, así como la expansión de conocimiento, destrezas y habilidades, que trabajan con el acceso de manera autónoma y universal debido a la utilización de teléfonos celulares compactos.

\section{Herramientas de aprendizaje social (Social Learning tools)}

También denominados "aparatos de aprendizaje social", se consideran instrumentos de aprendizaje amigables en el caso de que se hayan convertido en los activos utilizados en la medida de aprendizaje educativo con fines andragógicos e instructivos, supervisando la programación social o los medios basados en la web decididos a trabajar con el aprendizaje. en la comunicación entre individuos y marcos (Sánchez \& Pinochet, 2017).

Por otro lado, Jubany i Vila (2012) Sostiene que, en estos días, las administraciones de organizaciones avanzadas permiten que las personas que son esenciales para ellas, sus conocimientos y sus esfuerzos coordinados sean divulgados de manera básica desde cualquier lugar y cuando sea, intercambiar datos, pensamientos y experiencias garantizando su propio y experto desarrollo, permitiendo la producción de marcos de correspondencia significativos y unión social.

Para el creador, hay dos opciones para que las organizaciones ingresen a la interacción de exhibición y con qué sistemas, una alternativa es dejarlos fuera del divisor del salón principal sin explotar su capacidad instructiva de la utilización pedante de los PDA. La otra alternativa es aprovechar la forma en que los suplentes son ahora parte de ellos, se registran las asociaciones, se trabaja con la valoración de ciclos y la identificación de circunstancias conflictivas fuera de la organización. Por otra parte, González (2014) comunica que, las organizaciones interpersonales y los edublogs como dispositivos de aprendizaje sinérgico han mostrado nuevas condiciones virtuales para el aprendizaje utilizando avances dinámicos, la web como la increíble maravilla sociocultural y la mejora de la web, junto con una variedad de aparatos, han abierto un gran número de oportunidades para diversos puntos de vista como la preparación, la utilización de modelos constructivistas en los modelos de educación y aprendizaje, las medidas de desarrollo y mejora, al igual que en el aprendizaje comunitario (Chiquinquirá \& Hernández, 2020). 
En ese orden de ideas, Montero \& Gewerc (2015) han decidido en sus exámenes que la utilización de una organización informal partía de la necesidad de descubrir artilugios que consolidaran las partes sociales y colectivas del aprendizaje con el desarrollo que cada sujeto realiza en las condiciones individuales con las que aprende la persona en cuestión. Piensan que el desarrollo social de la idea de juventud y de las capacidades que necesitan los jóvenes para adaptarse a la nueva economía de la información también se utiliza como atractivo para incitar cambios instructivos.

Como lo indica la valoración de los creadores, con el argumento de partir de los requerimientos y supuestos trabajados en su interacción de la socialización con los avances, se debe proponer un cambio instructivo significativo, tanto en el programa educativo como en las instituciones educativas. Los nuevos avances se han convertido en otra situación instructiva que proyecta los resultados concebibles que ofrece la web y estimula al estudiante para que sea dinámico en el ciclo de aprendizaje interactuando con un área local de aprendizaje virtual (Carneiro, Toscano, \& Díaz, 2021).

En este punto de vista, Amaro (2020) piensa que el aprendizaje colectivo y la utilización de la innovación son dos patrones que están imperando en cada una de las fases instructivas de la persona, es decir, desde la etapa esencial hasta la universitaria, descubriendo un gran número de usos, escenarios virtuales que se están llevando a cabo de forma dinamita, mejorando a un ritmo desconcertante para tener la opción de conseguir contenidos característicos de una asignatura, al tiempo que se avanza en la cooperación entre educadores, alumnos suplentes como esfuerzo conjunto entre alumnos suplentes (Chiquinquirá \& Hernández, 2020).

En otro orden de ideas, Sánchez \& Pinochet (2017), hacen referencia a que el interés por las organizaciones informales no radica tanto en la sustancia como en su capacidad para construir asociaciones y sumar estima, ya que es la importancia en desarrollo de las interconexiones. Hubs a pesar de la información individualizada. Para este creador, el término e-learning se utiliza para aludir al descubrimiento que se comparte y se trabaja con los estudiantes reales que combinan y rediseñan la apropiación de la sustancia, adquiriendo habilidades computarizadas y cumpliendo con los objetivos de preparación.

Para el creador, cuando el e-learning se incorpora en un marco o etapas de aprendizaje de los ejecutivos, se puede abrir un entorno avanzado recibiendo cualidades de disponibilidad, construyendo asociaciones con marcos externos, mediante mashups, que es lo que encierra lo innovador y la interacción de recombinar los datos existentes y crear nuevos datos con esa información el sindicato insta a los clientes a establecer asociaciones entre el contenido de los medios dispersos, creando y encontrando nuevos tipos de 
datos que se muestran en varias organizaciones y espacios (González \& Medina, 2018).

Continúan Sánchez \& Pinochet (2017) expresando que mostrar el aprendizaje post-elearning reemplaza la transmisión directa de datos instructores por un modelo abierto de información dependiente de organizaciones interrelacionadas, abierto a nuevas agendas de preparación y a todas las multitudes, siendo dinámico todo el tiempo, considerando los estilos psicológicos de los sujetos y el surtido de gadgets que ofrecen acceso a información multisensorial verbal, compuesta, visual y sonora, aclimatando a otro método instruccional ajustado a una correspondencia portátil, adaptable, no directa, ajustada a los intereses y necesidades de los aprendices.

Para Sánchez \& Pinochet (2017) los posibles mashups aplicados al entorno instructivo radica en la forma en que hacen concebible llegar a nuevas resoluciones o reconocer nuevas conexiones a través de la asociación y combinación de mucha información, de diversas fuentes, de una manera razonable, para lo cual existe son aparatos que dan un clima vivo que se puede coordinar desde universos virtuales para que el estudiante en un espacio similar pueda acceder a un show, un video o un wiki, por ejemplo, Active Worlds, Olive y Second Life, permiten la colaboración en el uso de estos recursos.

En la sociedad actual, la verdad instructiva está firmemente conectada a los avances y desarrollos mecánicos desde el M-Learning o aprendizaje electrónico y el avance del aprendizaje electrónico versátil, ambos son concebibles utilizando etapas de PC, lo que aborda un beneficio; pues, la información en la práctica amistosa debe ser considerada por las diversas áreas de extraordinaria importancia. En consecuencia, la prueba actual para las tertulias comprometidas con este tema, solicita el cumplimiento de prerrequisitos empresariales combinados con la preparación de un individuo con una preparación inigualable que incorpore la formación con implicaciones telemáticas, así como la utilización de etapas innovadoras a la transmisión y edad de información (Sánchez \& Pinochet, 2017)

Volviendo a lo anterior, los científicos coinciden en que la utilización del enfoque M-Learning o aprendizaje portátil, se suma mutuamente al binomio de fundamentos instructivos del público en general; ya que aportan respuestas a los problemas de la sociedad globalizada; similar; Del mismo modo, no solo se suma y reacciona al problema del espacio real, sino que se puede utilizar de manera efectiva a pesar de cualquier emergencia o posibilidad mundial, también brinda ventajas de mejora del tiempo, economía en los espacios reales, disminución de costos que comienzan desde las distancias. y sucesivos desarrollos de las partes mediadoras. Posteriormente, tanto el e-learning como el e-learning portátil ofrecen una ayuda significativa en el área de la instrucción, entre diferentes motivos, se trata de una ruta de 
vanguardia y exitosa para la era y transmisión de información que favorece el desarrollo de la sociedad actual de la Información y correspondencia (Chiquinquirá \& Hernández, 2020).

Por último, los analistas advierten que hay que tener la certeza de que el M-Learning no aborda ni un ápice un sustituto de la preparación convencional ya que ambos pueden coincidir consumadamente atendiendo a los requerimientos, además, dado el vertiginoso ascenso de nuevas innovaciones, muy bien Reconocer que los estudiantes conocen bien los medios distintivos de PC, pero los instructores (educadores, entrenadores y facilitadores, entre otros) son las personas que manejan la innovación intelectual, además, su objetivo principal es mostrarles cómo actualizar y utilizar dichos teléfonos celulares o instrumentos, sin embargo con fines escolares posteriormente será la prueba que los educadores deben afrontar (Sánchez \& Pinochet, 2017).

\section{Método}

Como metodología de la investigación se desarrolló un estudio con enfoque cualitativo debido al análisis e interpretación de los resultados en función a la percepción de los actores activos (estudiantes) en el proceso de aprendizaje. En consecuencia mediante un diseño no experimental, método inductivo que se enfoca en analizar el fenómeno del objeto de estudio por medio de un alcance descriptivo que establece una evaluación del uso del aplicativo con base a las siguientes descriptores (Burden \& Kearney, 2017) (Ver Anexo 1):

- Pertinencia

- Facilidad de uso

- Personalización

- Retroalimentación

- Autenticidad

- Habilidades del pensamiento

- Trabajo colaborativo

- Motivación

Para el análisis de la información se procede al envió de la encuesta (formulario) hacia los estudiantes, esta encuesta está estructurada de acuerdo al instrumento proporcionado por autores Bedregal y Cornejo (2019) en la investigación titulado "Evaluación de la percepción estudiantil en relación al uso de la plataforma Moodle desde la perspectiva del TAM", además para la adaptación de los ítems para que los estudiantes sean capaces de comprender e interpretar los lineamientos y así mismo puedan seleccionar la respuesta que verdaderamente represente lo que ellos piensen. Dicho instrumento (anexo 2) 
presenta un Índice de Validación de Contenido (IVC) de 1,00 (Rozas \& Rojas, 2016).

Para el desarrollo de la recolección de información se tomó como unidad de análisis a los estudiantes de un Instituto Superior Tecnológico localizado en la ciudad de Daule, provincia del Guayas en Ecuador. Esta institución de educación superior se caracteriza por contar con una oferta de carreras amplia de diez carreras alineadas a las ciencias administrativas, informática, técnicas y tecnológicas tanto en modalidades presenciales como del sistema dual (50\% clases y $50 \%$ en empresas formadoras) con una población de 1408 estudiantes matriculados en el II periodo académico 20202021 (anexo 3), en consecuencia, por medio de un muestreo probabilístico aleatorio simple se calculó una muestra de 302 estudiantes. Así mismo la técnica e instrumento de recolección de información utilizado fue la encuesta por medio de Google formularios (anexo 3), cuyos ítems fueron evaluados por una escala de Likert donde los encuestados valoraron cada descriptor de imperceptible a fuerte (1-5).

Como se denota en el párrafo anterior, la unidad de análisis son los estudiantes de la institución de educación superior y es relevante también recalcar que se tomó este caso de estudio con base a la problemática provocada por la crisis sanitaria que todo el mundo está pasando por la pandemia y el contagio del SARS-CoV-2, todas las instituciones educativas ya sea inicial, media y superior se encuentran en modalidad virtual. Sin embargo, la institución ya lleva varios años utilizando plataformas virtuales, así mismo el uso de aplicativos móviles fue determinante para sostener este proceso académico ya que la institución al ser pública y estar ubicada en una zona rural la mayoría de sus estudiantes son de escasos recursos y no cuentan con una infraestructura tecnológica adecuada. Por tanto, la opción de una aplicación móvil fue muy atractiva ya que por medio de smartphones los estudiantes pueden realizar trabajos autónomos, presentar tareas y rendir tanto lecciones como exámenes.

\section{Análisis de resultados}

En función con la encuesta realizada a los estudiantes esto se lo ha clasificado en función a las ocho dimensiones determinadas también como descriptores y lo que se busca es identificar y analizar la intensidad y perspectiva de estos actores en relación con las características virtuales pedagógicas con respecto a las experiencias con la aplicación móvil utilizada en la institución. 
Tabla 2. Presentación de resultados

\begin{tabular}{|c|c|c|c|c|c|c|}
\hline \multirow{3}{*}{$\mathrm{N}^{\circ}-$} & \multirow{3}{*}{ ítem } & \multicolumn{5}{|c|}{ Imperceptible } \\
\hline & & \multicolumn{5}{|c|}{ Fuerte) } \\
\hline & & 1 & 2 & 3 & 4 & 5 \\
\hline 1 & $\begin{array}{c}\text { ¿Qué tan adaptables es el } \\
\text { aplicativo móvil para el } \\
\text { aprendizaje en relación con las } \\
\text { necesidades económicas de } \\
\text { estudiantes? }\end{array}$ & $2,60 \%$ & $3,60 \%$ & $27,20 \%$ & $40,40 \%$ & $26,20 \%$ \\
\hline 2 & $\begin{array}{l}\text { ¿Usted considera que todos los } \\
\text { estudiantes fueron consultados en } \\
\text { cuanto si cuentan o no con un } \\
\text { dispositivo móvil? }\end{array}$ & $43,60 \%$ & $39,40 \%$ & $4,00 \%$ & $3,30 \%$ & $9,70 \%$ \\
\hline 3 & $\begin{array}{l}\text { ¿Qué tan fácil es el uso del } \\
\text { aplicativo móvil para el } \\
\text { aprendizaje con respecto a las } \\
\text { gráficas, enlaces, navegación e } \\
\text { intuitivo? }\end{array}$ & $3,30 \%$ & $7,30 \%$ & $21,50 \%$ & $39,40 \%$ & $28,50 \%$ \\
\hline 4 & $\begin{array}{c}\text { ¿Usted considera que la interfaz } \\
\text { de la aplicación móvil } \\
\text { implementada por la institución } \\
\text { pueden provocar la } \\
\text { desconcentración del estudiante? }\end{array}$ & $26,20 \%$ & $10,60 \%$ & $6,00 \%$ & $43,00 \%$ & $14,20 \%$ \\
\hline 5 & $\begin{array}{l}\text { ¿Qué tan personalizado es el } \\
\text { aplicativo móvil en relación con } \\
\text { la necesidad de los estudiantes? }\end{array}$ & $3,30 \%$ & $4,00 \%$ & $18,90 \%$ & $43,00 \%$ & $30,80 \%$ \\
\hline 6 & $\begin{array}{c}\text { ¿Usted considera que el } \\
\text { diagnóstico realizado por la } \\
\text { institución en cuanto a la } \\
\text { capacidad de su dispositivo móvil } \\
\text { puede soportar el aplicativo para } \\
\text { un aprendizaje efectivo fue el } \\
\text { apropiado? } \\
\end{array}$ & $27,90 \%$ & $57,30 \%$ & $7,30 \%$ & $2,60 \%$ & $4,90 \%$ \\
\hline 7 & $\begin{array}{c}\text { ¿Qué tanto el aplicativo móvil } \\
\text { ofrece a los estudiantes una } \\
\text { retroalimentación específica y } \\
\text { personalizada? }\end{array}$ & $3,00 \%$ & $6,00 \%$ & $26,80 \%$ & $39,70 \%$ & $24,50 \%$ \\
\hline 8 & $\begin{array}{l}\text { ¿Usted considera que actualmente } \\
\text { tiene la capacidad de manejar } \\
\text { correctamente las funciones del } \\
\text { aplicativo móvil de la institución? }\end{array}$ & $7,60 \%$ & $3,30 \%$ & $46,40 \%$ & $17,90 \%$ & $21,50 \%$ \\
\hline 9 & $\begin{array}{l}\text { ¿Qué tanto el aplicativo móvil } \\
\text { permite desarrollar habilidades a } \\
\text { través de actividades de la vida } \\
\text { real en entornos auténticos y } \\
\text { basados en el contexto del } \\
\text { estudiante? }\end{array}$ & $1,70 \%$ & $6,60 \%$ & $24,80 \%$ & $43,40 \%$ & $23,50 \%$ \\
\hline
\end{tabular}




\begin{tabular}{|c|c|c|c|c|c|c|}
\hline 10 & $\begin{array}{l}\text { ¿Usted considera que el } \\
\text { aplicativo móvil puede suplir de } \\
\text { gran manera la interacción entre } \\
\text { docente y estudiante en el ámbito } \\
\text { de compartir experiencias a nivel } \\
\text { profesional de forma efectiva? }\end{array}$ & $3,00 \%$ & $7,70 \%$ & $25,10 \%$ & $39,70 \%$ & $24,50 \%$ \\
\hline 11 & $\begin{array}{l}\text { ¿Qué tanto el aplicativo móvil } \\
\text { promueve el desarrollo de } \\
\text { habilidades de pensamiento de } \\
\text { orden superior, creación, } \\
\text { evaluación y análisis? }\end{array}$ & $2,00 \%$ & $4,60 \%$ & $22,80 \%$ & $43,40 \%$ & $27,20 \%$ \\
\hline 12 & $\begin{array}{c}\text { ¿Usted considera que los } \\
\text { contenidos que se imparten en los } \\
\text { aplicativos móviles fueron lo } \\
\text { suficientemente adaptados a la } \\
\text { virtualidad? }\end{array}$ & $51,60 \%$ & $31,70 \%$ & $9,80 \%$ & $2,00 \%$ & $4,90 \%$ \\
\hline 13 & $\begin{array}{l}\text { ¿Qué tanto el aplicativo móvil } \\
\text { fomenta la comunicación entre } \\
\text { los estudiantes, la } \\
\text { creación/modificación del } \\
\text { contenido de forma colaborativa } \\
\text { y facilidad al compartir dicho } \\
\text { contenido online? }\end{array}$ & $3,60 \%$ & $5,30 \%$ & $26,80 \%$ & $39,10 \%$ & $25,20 \%$ \\
\hline 14 & $\begin{array}{c}\text { ¿Usted considera que la } \\
\text { aplicación móvil generó una } \\
\text { barrera de comunicación } \\
\text { intrapersonal con sus compañeros } \\
\text { de curso? }\end{array}$ & $24,80 \%$ & $31,70 \%$ & $28,40 \%$ & $8,20 \%$ & $6,90 \%$ \\
\hline 15 & $\begin{array}{l}\text { ¿Qué tan motivado te sientes para } \\
\text { usar el aplicativo móvil y elegir } \\
\text { entre otras opciones similares? }\end{array}$ & $3,00 \%$ & $5,30 \%$ & $24,80 \%$ & $37,40 \%$ & $29,50 \%$ \\
\hline 16 & $\begin{array}{l}\text { ¿Usted considera haber percibido } \\
\text { frustración cuando el aplicativo } \\
\text { móvil o su dispositivo no podían } \\
\text { ofrecerle un acceso optimo a la } \\
\text { clase? }\end{array}$ & $59,30 \%$ & $31,70 \%$ & $5,00 \%$ & $2,00 \%$ & $2,00 \%$ \\
\hline
\end{tabular}

Pertinencia

Este instrumento ofrece dos perspectivas con respecto a la usabilidad del aplicativo móvil implementado en la institución de educación superior desde un visión que evaluó los atributos y otra que trata de identificar las barreras que impiden el desarrollo del proceso de aprendizaje por medio del aplicativo. En relación con la pertinencia los aplicativos móviles es justificado por la necesidad de que el futuro profesional adquiera competencias como razonamiento, capacidad de relacionarse e integrar conocimientos, así como trabajar en equipo (Serrano, 2019). En consecuencia, se logró identificar que en cuanto a la adaptabilidad de este instrumento tecnológico en relación con las necesidades existe una fuerte aceptación por parte de los estudiantes 
encuestados (nivel $4=40,4 \%$ y $5=26,2 \%$ ). sin embargo, no todos los estudiantes fueron consultados en cuanto si cuentan o no con un dispositivo móvil generando la imperceptibilidad de ellos (nivel $1=43,6 \%$ y $2=39,4 \%$ ).

Facilidad de uso

Por otra parte, la necesidad de introducir un instrumento utilitario en la escolarización y dentro del mercado laboral surgió debido a la exigencia de fundamentos instructivos para llevar a cabo avances de aprendizaje e información (TAC) como dispositivos de instrucción para reforzar la información y recrear circunstancias con conexión con el mundo real (Guadarrama, 2021). Por lo tanto, la facilidad que genera el aplicativo por el uso de gráficas, enlaces y navegación obtuvo una fuerte aceptación por parte de los encuestados logrando las puntuaciones más altas en los tres niveles considerados fuertes (nivel $3=21,5 \%, 4=39,4 \%$ y $5=28,5 \%$ ). Pero, en cuanto a la concentración de los estudiantes existe un porcentaje considerable que señala la ocurrencia de tal situación como fuerte (nivel $4=43 \%$ y $5=14,2 \%$ ).

Personalización

Así mismo, en el desarrollo de aplicativos móviles versátiles, se utiliza la personalización de contenido, cuyo objetivo es mantenerse alejado de la sobrecarga de datos (infoxicación), ajustando la sustancia particular para cada tipo de estudiante, ya sea un individuo o una organización. La combinación de dispositivos y avances adecuados permite atender la variedad de puntos y beneficiarios, incluso utilizando herramientas externas que trabajan con la evaluación evolutiva y sumativa a través de la comunicación directa (Trabaldo, Soraide, \& Kamijo, 2017). Así mismo, la personalización del aplicativo móvil en relación con las necesidades de los estudiantes fue valorado como fuerte debido a que la mayoría de los encuestados seleccionaron los dos niveles más fuertes (nivel $4=43 \%$ y $5=30,8 \%$ ). Por otro lado, considerando el diagnóstico realizado por la institución en cuanto a la capacidad del dispositivo móvil en soportar el aplicativo fue determinado como imperceptible debido a la selección mayoritaria en los niveles menos fuertes (nivel $1=27,9 \%, 2=57,3 \%$ ).

\section{Retroalimentación}

La creación de aplicaciones portátiles cruzadas para el refuerzo de tipos recíprocos de instrucción es una posible respuesta para la mejora continua del aprendizaje en estudiantes suplentes que están aprendiendo en la universidad $o$ en cualquier nivel más significativo. Los insumos en aplicaciones versátiles se utilizan como ayuda en varias clases, aceptando las nuevas dificultades de hoy y de esta manera ofrecer algunas críticas dependientes del módulo Feedback que propondrá datos tomados de las 
calificaciones evaluadas y con esto dará nuevos dispositivos utilizando la innovación actual (Santos, Hernández, Chau, Zepahua, \& Torres, 2017). Según los estudiantes encuestados aprueban con una calificación fuerte sobre la retroalimentación de las clases desarrolladas en entornos virtuales se ajusta a los requerimientos temporales y tecnológicos de ellos con porcentajes significativos en los niveles más fuertes (nivel $4=39,7 \%$ y 24,5 \%). Además, se evaluó la capacidad de que tienen los estudiantes en el manejo correcto de las funciones del aplicativo móvil cuyos resultados marcan una imparcialidad debido a que el $46,4 \%$ de los encuestados seleccionaron el nivel 3 (nivel neutro).

\section{Autenticidad}

Los teléfonos celulares se utilizan en la instrucción como árbitros en la medida de instrucción y aprendizaje, dado que estos dispositivos se pueden utilizar para asesorar a diferentes materiales instructivos, esto implicaría que el uso debería promover la mejora de las habilidades asociadas con la tarea de dominio. así como ayudar a mejorar las habilidades de razonamiento y ayudar a los amigos, los teléfonos celulares pueden expandir la inspiración de los estudiantes dentro de la sala de estudio (Rodríguez \& Juárez). Los estudiantes fueron evaluados con respecto a la capacidad del aplicativo móvil en el desarrollo de habilidades a través de actividades de la vida real en entornos auténticos y los resultados mostraron que son fuertes dicha afirmación (nivel $4=43,4 \%$ y $5=23,5 \%$ ). Así mismo, los encuestados declaran que el dispositivo tiene una capacidad fuerte en suplir la interacción entre el docente y el estudiante en el ámbito de compartir experiencias que enriquece la perspectiva profesional del estudiante.

Habilidades del pensamiento

Las aplicaciones móviles totalmente destinadas a apoyar y acompañar al estudiante en el camino hacia la adquisición de conocimiento como también la actualización de los grados de información y el grado de habilidades básicas de razonamiento inteligente o de pensamiento, se centra en la mejora de los datos y la correspondencia de los activos de las TIC, coordinados hacia la preparación, la apertura y la conveniencia, consolidando modelos instructivos contemporáneos de tipo constructivista (Arias \& Constain, 2021). En función a la capacidad que tiene el aplicativo móvil en el desarrollo de habilidades de pensamiento de orden superior, creación, evaluación y análisis los estudiantes encuestados señalan la gran mayoría como fuerte debido a que han seleccionado los niveles más fuertes (nivel $3=22,8 \%, 4=43,4 \%$ y $27,2 \%$ ). Sin embargo, en cuanto a la adaptación de los contenidos en el aplicativo móvil fueron débiles o imperceptibles por parte de los estudiantes señalando 
la mayoría en los niveles menos fuertes (nivel $1=51,6 \%, 2=31,7 \%$ y $3=$ $9,8 \%)$.

\section{Trabajo colaborativo}

Una columna significativa en los ciclos y espacios instructivos es el esfuerzo coordinado, que aprovecha las TIC, las personas que interceden en una interacción de aprendizaje, comúnmente se influyen, intercambian emprendimientos y supuestos y reconsideran un emprendimiento compartido que los impulsa a la realización compartida de otra titulación. de información y cumplimiento (Gil, 2019). Por tanto, según los estudiantes encuestados describen como fuerte en que el aplicativo móvil fomenta la comunicación entre los estudiantes, la creación/modificación del contenido de forma colaborativa y facilidad al compartir dicho contenido online (nivel $4=39,1 \%$ y nivel $5=25,2 \%$ ). por otro lado, los encuestados consideran imperceptible que la aplicación móvil generó una barrera de comunicación intrapersonal con sus compañeros de curso (nivel $1=24,8 \%, 2=31,7 \%$ y $3=28,4 \%$ ).

\section{Motivación}

Finalmente, los procedimientos instructivos son en conjunto los activos que se aplican para trabajar con el aprendizaje de estudiantes suplentes, los que se identifican con la motivación son aquellos que significan revitalizar la información pasada e incrementar los grados de actitud de los estudiantes antes de una reunión de aprendizaje (Estrada, 2017). Así mismo se evaluó por parte de los estudiantes una motivación fuerte (nivel $4=37,4 \%$ y $5=29,5 \%$ ) en el uso del aplicativo móvil como también la elección de elegir entre otras opciones similares. sin embargo, durante el ejercicio de las clases la mayoría de los estudiantes han percibido frustración debido a la conectividad o al colapso del aplicativo por tanto, una gran proporción de estudiantes seleccionar los niveles menos fuertes (nivel $1=59,3 \%$ y $2=31,7 \%$ ).

\section{Conclusiones}

En este estudio se han analizado diferentes perspectivas en cuanto a la usabilidad y la integración de la tecnológica en el desarrollo del aprendizaje desde la perspectiva de los estudiantes los cuales evaluaron las ventajas y desventajas al implementar un aplicativo móvil en el proceso de aprendizaje en una institución de educación superior técnica y tecnológica. Con este enfoque se puede describir que los reajustes en el diseño es un proceso que no se lo debe tomar a la ligera, la correcta aplicación mediante metodologías correctivas y retroalimentaciones de las experiencias de los usuarios evita poner a disposición de estos productos tecnológicos de aprendizaje de mala calidad. Por tanto, la evaluación periódica de la usabilidad es un proceso que además de visibilizar los distintos errores, se convierte en una herramienta de 
control de calidad que permite desarrollar aplicaciones web confiables y usables. No obstante la evaluación comparativa entre ventajas y desventajas permite determinar barreras en la interfaz con mayor presencia en cada una de las dimensiones estudiadas, permitiendo así identificar las áreas que necesitan mayor atención.

El proceso de hacer usable a una herramienta tecnológica como las aplicaciones web no es una tarea sencilla, requiere de la asociación entre el contenido, la interacción y los procesos. Un contenido de calidad debe tratar de ser concreto y resumir al máximo su contenido. La interacción debe estar en función del público objetivo. Los procesos deben ayudar a encontrar una lógica en la interacción. La armonía de estos elementos más el monitoreo y la medición constante de los elementos ayudaran a evitar una percepción negativa por parte de los usuarios.

Por tanto la usabilidad hará de las aplicaciones web lugares de mayor tránsito donde no existan limitantes según el nivel de conocimiento de cada usuario, por lo contrario esta disciplina abrirá la posibilidad de desarrollar nuevos conceptos y teorías que a futuro puedan aportar a otras instituciones educativas a difundir el tema y a ampliar campos educativo, profesional y social con muchas posibilidades y aristas para su expansión y desarrollo a nivel de calidad en el aprendizaje.

La facilidad de uso se convierte en un tema básico para el reconocimiento de una aplicación, es así como la óptica tecnológica del estudiante permite extender el examen del plan centrado en el usuario, dando entrada al nivel de atributos relacionados con la calidad de programación. Las cualidades atribuidas a una aplicación son ideas dinámicas, debido a esto, no se pueden estimar directamente, para medirlas es importante relacionar varias medidas, por ejemplo, la característica o directriz, la eficiencia se puede evaluar utilizando una métrica que permite calcular el tiempo que tarda un estudiante en terminar una actividad establecida.

Sin embargo, existen grandes barreras que las instituciones de educación deben de considerar como la capacidad económica en la adquisición de dispositivos móviles, como también en el acceso óptimo a internet para el desarrollo de las clases. Además, la continuidad de estas dificultades genera retrocesos en el aprendizaje de los estudiantes creando frustración y la posible deserción estudiantil. Por tanto, es necesario que la institución realice un riguroso diagnóstico de la situación a nivel tecnológico de los estudiantes para establecer un aplicativo $\mathrm{u}$ otro medio a nivel tecnológico que aporte significativamente el desarrollo del proceso educativo.

Por lo tanto, los tipos de trabajo y los recursos utilizados serían ilimitados: desde abordar diversas reacciones, ejercicios de respuesta corta, ejercicios instructivos explícitos como examen, donde no solo el educador podría enviar tareas, contenido, señala y corrige los ejercicios, pero además 
los estudiantes pueden cooperar en los remedios y presentar contenido de medios mixtos para toda la clase.

\section{References:}

1. Aguiar, B., Velázquez, R., \& Aguiar, J. (2019). Innovación docente y empleo de las TIC en la Educación Superior. Espacios.

2. Arias, J., \& Constain, G. (2021). Experiencia de diseño de aplicaciones móviles basada en estrategias de gamificación para el fortalecimiento de habilidades cognitivas. Revista de la Asociación Interacción Persona Ordenador (AIPO), 2(1), 17-24.

3. Burden, K., \& Kearney, M. (2017). Investigating and critiquing teacher educators' mobile learning practices. Interactive Technology and Smart Education. doi:10.1108/ITSE-05-2017-0027

4. Carneiro, R., Toscano, J., \& Díaz, T. (2021). Los desafíos de las TIC para el cambio educativo. Fundación Santillana.

5. Castro, S., \& Guzmán, B. (2007). Las Tic en los procesos de enseñanza y aprendizaje. Revista de Educación Laurus, 213-234.

6. Cavaller, V. (2016). La tecnología cognitiva. Recuperado de https://n9.cl/6yegk.

7. Chiquinquirá, R., \& Hernández, J. (2020). M-Learning: Estrategia Para La Promoción Del Aprendizaje Electrónico Móvil En Instituciones De Educación Superior. Universidad Privada Dr. Rafael Belloso Chacín.

8. Coll, C., \& Monereo, C. (2008). Psicología de la educación virtual. Ediciones Morata.

9. Cruz, A., Aguilar, A., Colomo, E., Gabarda, E., \& Domínguez, R. (2018). Propuesta Multidiciplinares de innovación e intervención educativa. Universidad Internacional de Valencia.

10. Estrada, C. (2017). strategias Educativas De Motivación A Través De Una Aplicación Móvil Para Evocación De Saberes Previos En Estudiantes De Ofimática De Zegel Ipae. Universidad César Vallejo.

11. Gallegos, J. (2015). Las Prácticas Pedagógicas que realizan los asistentes de la educación, durante los recreos en los patios de la escuela públicas Básicas de Valparaíso y su relevancia en la gestión de la convivencia. Universidad Autónoma de Barcelona, 22-48.

12. García, R., \& Corona, J. (2020). M-learning: strategy for the promotion of mobile e-learning in higher education institutions. Universidad Privada Dr. Rafael Belloso Chacín.

13. Gewer, A., \& Montero, L. (2015). Colaboración y redes sociales en la enseñanza universitaria. Revista Científica de Comunicación y Educación. 
14. Gil, J. (2019). Interconectados apostando por la construcción colectiva del conocimiento Aprendizaje móvil en educación infantil y primaria. Píxel-Bit: Revista de Medios y Educación, 54, 185-203.

15. González, D. (2017). Ambientes colaborativos virtuales para el aprendizaje individual. Universidad de Costa Rica, 1-29.

16. González, R., \& Medina, G. (2018). Uso de dispositivo móviles como herramientas para aprender. Pixel-Bit: Revista de Medios y Educación, 217-227.

17. Guadarrama, J. (2021). Usabilidad de una app móvil para el aprendizaje y elaboración de estados financieros en México. RIDE Revista Iberoamericana para la Investigación y el Desarrollo Educativo, 11(22).

18. Hernández, R., \& Morales, M. (2010). Dispositivos móviles en educación. Revista América Learning \& Media. Recuperado de https://n9.cl/ae16e.

19. Herrera, B. (2012). El móvil en la educación: Un nuevo paradigma. Ventajas y desventajas de su uso. Recuperado de https://n9.cl/hxw5m.

20. Herrera, M. (2018). El refuerzo pedagógico como herramienta para el mejoramiento de los. Universidad Andina Simón Bolívar, 17-30.

21. Jubany i Vila, J. (2012). Sistema de Información Científica. Pixel-Bit. Revista de Medios y Educación, 239-240.

22. Landon, b. (1989). online educational delivery Applications: a web tool for comparative analysis. Obtenido de http://www.c2t2.ca/landonline/

23. Marqués, P. (2013). Claves para mejorar los aprendizajes integrando las tecnologías móviles en las clases" en Tecnología móvil e innovación en el aula. Nuevos retos y realidades educativas. Universidad de La Rioja. Recuperado de https://n9.cl/aulz.

24. Mauri, T., Onrubia, J., Coll, C., \& Colomina, R. (2016). La calidad de los contenidos educativos reutilizables: diseño, usabilidad y prácticas de uso. Revista de Educación a distancia, 50, 1-9.

25. Mujica, R. (2020). E-Learning como estrategia pedagógica en la educación superior. Revista Tecnológica Educativa Docentes.

26. Naranjo, E. C. (2019). Estrategia de formación virtual basada en el modelo ADDIE para fortalecer competencias pedagógicas y tecnológicas de los docentes del colegio WESLEYANO NORTE. Universidad EAN, 30-42.

27. Organización Mundial del Comercio. (2018). El futuro del comercio mundial: cómo las tecnologías digitales están transformando el comercio mundial. Organización Mundial del Comercio.

28. Ortiz, A., Colón, J., \& Agreda, M. (2018). Gamificación en educación: una panorámica sobre el estado de la cuestión. Scielo, 2-5. 
29. Pérez-Berenguer, d., \& García-Molina, J. (2016). Un enfoque para la creación de contenido online interactivo en las instituciones universitarias. Revista Educación a distancia, 51, 1-24.

30. Pujals, P. (2018). Modelo de comunicación para la enseñanza a distancia en internet. Universidad Autónoma de Barcelona, 14-31.

31. Rincón, A. (2016). Practicas innovadoras de investigación educativa de TIC que posibilitan el desarrollo profesional docente. Universidad Autónoma de Barcelona, 31-36.

32. Rodríguez, E. (2018). Importancia del manejo de competencias tecnológicas en las prácticas docentes de la Universidad Nacional Experimental de la Seguridad (UNES). Universidad De Costa Rica, 25.

33. Rodríguez, J., \& Juárez, J. (s.f.). Impacto del m-learning en el proceso de aprendizaje: habilidades y conocimiento. RIDE. Revista Iberoamericana para la Investigación y el Desarrollo Educativo.

34. Sánchez, M., \& Pinochet, G. (2017). El rol de las redes sociales virtuales en la difusión de información y conocimiento: estudio de casos. https://www.redalyc.org/revista.oa? id=1872, 107-135.

35. Santos, S., Hernández, L., Chau, A., Zepahua, B., \& Torres, C. (2017). Aplicación web e-learning multiplataforma para recolección de datos de usuarios y retroalimentación automática basada en técnicas estadísticas. Res. Comput. Sci., 135, 9-23.

36. Serrano, E. (2019). Modelo de Aprendizaje Basado en Casos y Problemas mediante la Integración de Capsulas de Autoaprendizaje y Aplicaciones Móviles Instrumentales en la Formación del Personal de Salud (CAPPS). Revista de Ciències de l'Educació, 1(2), 43-51.

37. Trabaldo, S., Soraide, M., \& Kamijo, M. (2017). Diseño y desarrollo de materiales de aprendizaje personalizados en dispositivos móviles para diferentes asignaturas. In IV Jornadas de TIC e Innovación en el Aula.

38. UNESCO. (2019). Recomendación sobre los Recursos Educativos Abiertos (REA). Organización de las Naciones Unidas para la Educación, la Ciencia, y la Cultura, 1-5.

39. Valencia, N., huertas, A., \& Baracaldo, P. (2014). Virtual learning environments: review of publications between 2003-2013 from the evidence-based pedagogy perspective. Revista Colombiana de Educación, 66, 73-103. 


\section{ANEXOS}

Anexo 1: Descriptores

\begin{tabular}{|c|c|c|c|c|}
\hline \multicolumn{5}{|c|}{ Rúbrica para evaluar la calidad de apps educativas } \\
\hline \multicolumn{5}{|c|}{ Nombre de la aplicación móvil ___ } \\
\hline \multirow{2}{*}{$\begin{array}{l}\text { DESCRIPTORES / } \\
\text { VALORACIÓN }\end{array}$} & EXCELENTE & BUENO & REGULAR & DEFICIENTE \\
\hline & 4 & 3 & 2 & 1 \\
\hline Pertinencia & \begin{tabular}{|c|}
$\begin{array}{c}\text { La app está estrechamente relacionada con el } \\
\text { propósito para el cual fue creada y es adecuada } \\
\text { para el estudiante }\end{array}$ \\
\end{tabular} & \begin{tabular}{|c|}
$\begin{array}{c}\text { La app está relacionada con el propósito } \\
\text { para el cual fue creada y es, en gran parte, } \\
\text { adecuada para el estudiante }\end{array}$ \\
\end{tabular} & \begin{tabular}{|c|} 
La app está poco relacionada con el \\
proposito para el cual fue creada puede no \\
ser adecuada para el estudiante
\end{tabular} & \begin{tabular}{|c|}
$\begin{array}{c}\text { La app no está relacionada con el propósito } \\
\text { para el cual fue creada ni tampoco es } \\
\text { adecuada para el estudiante }\end{array}$ \\
\end{tabular} \\
\hline Facilidad de uso & $\begin{array}{l}\text { Los gráficos y enlaces son muy adecuados y la } \\
\text { navegación es muy fáci. El uso de la app es } \\
\text { muy intulitio }\end{array}$ & \begin{tabular}{|c|} 
Los gráficos y enlaces son adecuados y la \\
navegación es fícil aunque aprender a usar \\
la app puede demandar cierto tiempo
\end{tabular} & 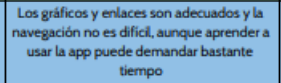 & \begin{tabular}{|l|} 
Los gráficos y enlaces son pobres $y$ la \\
navegacón es áficil.Aprender a usar la app \\
puede demandar mucho tiempo
\end{tabular} \\
\hline Personalización & \begin{tabular}{|c|} 
La app es completamente personalizable El \\
estudiante puede modificar la configuración y \\
las preferencias para ajustarla a sus \\
necesidades
\end{tabular} & $\begin{array}{l}\text { La app es personalizable. El estudiante } \\
\text { puede modilicar varios aspectos de la } \\
\text { configuración y de las preferencias para } \\
\text { ajustarla a sus necesidades }\end{array}$ & \begin{tabular}{|c|} 
La app es parcialmente personalizable. El \\
estudiante puede modficar muy pocos \\
aspectos de la configuración y de las \\
preferencias para ajustarla a sus necesidades
\end{tabular} & \begin{tabular}{|c|}
$\begin{array}{c}\text { La app no es personslizable El estudiante } \\
\text { esta imposibilitado de modificar la } \\
\text { configuración y las preferencias }\end{array}$ \\
\end{tabular} \\
\hline Retroalimentación & $\begin{array}{c}\text { La app brinda al estudiante retroalimentación } \\
\text { especifica y personalizada }\end{array}$ & $\begin{array}{l}\text { La app brinda al estudiante } \\
\text { retroalimentación general }\end{array}$ & $\begin{array}{l}\text { La app brinda al estudiante } \\
\text { retroalimentación limitada }\end{array}$ & $\begin{array}{c}\text { La app no brinda al estudiante ningún tipo } \\
\text { de retroalimentación }\end{array}$ \\
\hline Autenticidad & $\begin{array}{c}\text { La app permite desarrollar habilidades a través } \\
\text { actividades de la vida real en entornos } \\
\text { auténticos y basados en el contexto del } \\
\text { estudiante }\end{array}$ & \begin{tabular}{|c|}
$\begin{array}{c}\text { Algunos aspectos de la app representan un } \\
\text { entomo de aprendizaje auténtico y basado } \\
\text { en el contexto del estudiante }\end{array}$ \\
\end{tabular} & $\begin{array}{l}\text { La app ofrece actividades y entornos de } \\
\text { aprendizaje que se desarrollan a modo de } \\
\text { juegos o simulaciones }\end{array}$ & $\begin{array}{c}\text { No hay actividades realistas y el entorno de } \\
\text { aprendizaje es artificial y no está relacionado } \\
\text { con la vida real }\end{array}$ \\
\hline $\begin{array}{l}\text { Habilidades de } \\
\text { pensamiento }\end{array}$ & \begin{tabular}{|c|}
$\begin{array}{c}\text { La app promueve el desamrollo de habilidades } \\
\text { de pensamiento de orden superior: creación, } \\
\text { evaluación, y andlisis }\end{array}$ \\
\end{tabular} & \begin{tabular}{|c|}
$\begin{array}{c}\text { La app permite el desarrollo de habilidades } \\
\text { de pensamiento de orden superior: } \\
\text { evaluacion, andilisis y aplicación }\end{array}$ \\
\end{tabular} & \begin{tabular}{|c|}
$\begin{array}{c}\text { La app permite el desarrollo de habilidades } \\
\text { de pensamiento de orden inferior: } \\
\text { comprensión y memorización }\end{array}$ \\
\end{tabular} & $\begin{array}{l}\text { La app es limitada en el desarrollo de } \\
\text { habilidades de pensamiento de orden } \\
\text { inferior. comprensión y memorización }\end{array}$ \\
\hline Trabajo colaborativo & $\begin{array}{c}\text { La app fomenta la comunicación entre los } \\
\text { estudiantes, la creación/modificación del } \\
\text { contenido de forma colaborativa, y facilita el } \\
\text { compartir dicho contenido online }\end{array}$ & \begin{tabular}{|c|}
$\begin{array}{c}\text { La app facilita la comunicacón entre los } \\
\text { estudiantes, permite crear/modificar el } \\
\text { contenido de forma colaborativa y facilita el } \\
\text { compartir dicho contenido online }\end{array}$ \\
\end{tabular} & $\begin{array}{l}\text { La app permite la comunicación y la } \\
\text { colaboración online de forma limitada }\end{array}$ & $\begin{array}{l}\text { La app no permite nirgún tipo de } \\
\text { comunicacón ni colaboración entre los } \\
\text { estudiantes }\end{array}$ \\
\hline Motivación & \begin{tabular}{|c|}
$\begin{array}{c}\text { El estudiante se siente muy motivado para usar } \\
\text { la app y la elige como primera opcón entre } \\
\text { otras apps similares }\end{array}$ \\
\end{tabular} & \begin{tabular}{|c|}
$\begin{array}{c}\text { El estudiante utiliza la aplicacón según las } \\
\text { indicaciones del docente }\end{array}$ \\
\end{tabular} & \begin{tabular}{|c|} 
El estudiante utiliza la app de manera \\
forzada y la considera como una tarea \\
escolar más. A menudo se distrae al utilizarla
\end{tabular} & \begin{tabular}{|c|}
$\begin{array}{c}\text { El estudiante evita el uso de la appo } \\
\text { expresa su descontento cuando el docente } \\
\text { le pide que la utilice }\end{array}$ \\
\end{tabular} \\
\hline
\end{tabular}


Anexo 2: Índice de validación de contenido

\begin{tabular}{|c|c|c|c|c|c|}
\hline \multirow[b]{2}{*}{$n^{\circ}-$} & \multirow[b]{2}{*}{ Ítems } & \multicolumn{4}{|c|}{ Jueces } \\
\hline & & $\begin{array}{l}\text { Ing. John } \\
\text { Tobar } \\
\text { Litardo, } \\
\text { Msc. PhD. }\end{array}$ & $\begin{array}{l}\text { Lcda. } \\
\text { Evelyn } \\
\text { Pinargote } \\
\text { Junco, Msc. } \\
\text { PhD. } \\
\end{array}$ & $\begin{array}{l}\text { Ing. Galo } \\
\text { Tobar } \\
\text { Farias, } \\
\text { Msc. PhD. }\end{array}$ & $\mathrm{RCV}$ \\
\hline 1 & $\begin{array}{l}\text { ¿Qué tan adaptables es el } \\
\text { aplicativo móvil para el } \\
\text { aprendizaje en relación con las } \\
\text { necesidades económicas de } \\
\text { estudiantes? }\end{array}$ & 3 & 3 & 4 & 1,0 \\
\hline 2 & $\begin{array}{l}\text { ¿Usted considera que todos los } \\
\text { estudiantes fueron consultados } \\
\text { en cuanto si cuentan o no con } \\
\text { un dispositivo móvil? }\end{array}$ & 1 & 3 & 4 & 0,7 \\
\hline 3 & $\begin{array}{l}\text { ¿Qué tan fácil es el uso del } \\
\text { aplicativo móvil para el } \\
\text { aprendizaje con respecto a las } \\
\text { gráficas, enlaces, navegación e } \\
\text { intuitivo? }\end{array}$ & 2 & 2 & 3 & 1,0 \\
\hline 4 & $\begin{array}{l}\text { ¿Usted considera que la interfaz } \\
\text { de la aplicación móvil } \\
\text { implementada por la institución } \\
\text { pueden provocar la } \\
\text { desconcentración del } \\
\text { estudiante? }\end{array}$ & 4 & 1 & 2 & 0,7 \\
\hline 5 & $\begin{array}{l}\text { ¿Qué tan personalizado es el } \\
\text { aplicativo móvil en relación con } \\
\text { la necesidad de los estudiantes? }\end{array}$ & 3 & 3 & 1 & 0,7 \\
\hline 6 & $\begin{array}{l}\text { ¿Usted considera que el } \\
\text { diagnóstico realizado por la } \\
\text { institución en cuanto a la } \\
\text { capacidad de su dispositivo } \\
\text { móvil puede soportar el } \\
\text { aplicativo para un aprendizaje } \\
\text { efectivo fue el apropiado? }\end{array}$ & 3 & 4 & 1 & 0,7 \\
\hline 7 & $\begin{array}{l}\text { ¿Qué tanto el aplicativo móvil } \\
\text { ofrece a los estudiantes una } \\
\text { retroalimentación específica y } \\
\text { personalizada? }\end{array}$ & 4 & 3 & 2 & 1,0 \\
\hline 8 & $\begin{array}{l}\text { ¿Usted considera que } \\
\text { actualmente tiene la capacidad } \\
\text { de manejar correctamente las } \\
\text { funciones del aplicativo móvil } \\
\text { de la institución? }\end{array}$ & 2 & 4 & 3 & 1,0 \\
\hline 9 & $\begin{array}{l}\text { ¿Qué tanto el aplicativo móvil } \\
\text { permite desarrollar habilidades } \\
\text { a través de actividades de la } \\
\text { vida real en entornos auténticos }\end{array}$ & 4 & 3 & 4 & 1,0 \\
\hline
\end{tabular}




\begin{tabular}{|c|c|c|c|c|c|}
\hline & $\begin{array}{l}\text { y basados en el contexto del } \\
\text { estudiante? }\end{array}$ & & & & \\
\hline 10 & $\begin{array}{c}\text { ¿Usted considera que el } \\
\text { aplicativo móvil puede suplir de } \\
\text { gran manera la interacción entre } \\
\text { docente y estudiante en el } \\
\text { ámbito de compartir } \\
\text { experiencias a nivel profesional } \\
\text { de forma efectiva? }\end{array}$ & 4 & 2 & 3 & 1,0 \\
\hline 11 & $\begin{array}{l}\text { ¿Qué tanto el aplicativo móvil } \\
\text { promueve el desarrollo de } \\
\text { habilidades de pensamiento de } \\
\text { orden superior, creación, } \\
\text { evaluación y análisis? }\end{array}$ & 3 & 3 & 2 & 1,0 \\
\hline 12 & $\begin{array}{c}\text { ¿Usted considera que los } \\
\text { contenidos que se imparten en } \\
\text { los aplicativos móviles fueron } \\
\text { lo suficientemente adaptados a } \\
\text { la virtualidad? }\end{array}$ & 3 & 3 & 3 & 1,0 \\
\hline 13 & $\begin{array}{l}\text { ¿Qué tanto el aplicativo móvil } \\
\text { fomenta la comunicación entre } \\
\text { los estudiantes, la } \\
\text { creación/modificación del } \\
\text { contenido de forma colaborativa } \\
\text { y fallecida el compartir dicho } \\
\text { contenido online? }\end{array}$ & 3 & 4 & 4 & 1,0 \\
\hline 14 & $\begin{array}{c}\text { ¿Usted considera que la } \\
\text { aplicación móvil generó una } \\
\text { barrera de comunicación } \\
\text { intrapersonal con sus } \\
\text { compañeros de curso? }\end{array}$ & 2 & 3 & 4 & 1,0 \\
\hline 15 & $\begin{array}{l}\text { ¿Qué tan motivado te sientes } \\
\text { para usar el aplicativo móvil y } \\
\text { elegir entre otras opciones } \\
\text { similares? }\end{array}$ & 3 & 4 & 4 & 1,0 \\
\hline 16 & $\begin{array}{c}\text { ¿Usted considera haber } \\
\text { percibido frustración cuando el } \\
\text { aplicativo móvil o su } \\
\text { dispositivo no podían ofrecerle } \\
\text { un acceso optimo a la clase? }\end{array}$ & 4 & 3 & 2 & 1,0 \\
\hline \multicolumn{2}{|c|}{ Razón de validez de contenido - RCV } & \multicolumn{4}{|c|}{$R V C^{\prime}=\frac{\text { Cantidad de jueces que puntuaron entre } 3 \text { y } 4 \text { al item }}{\text { Total de jueces }}$} \\
\hline \multicolumn{2}{|r|}{$\begin{array}{l}\text { Índice de validación de contenido - } \\
\text { IVC }\end{array}$} & \multicolumn{2}{|c|}{$I V C=\frac{\left.\sum_{i=1}^{M} \text { CVR (aceptables }\right)_{i}}{M}$} & \multicolumn{2}{|c|}{1} \\
\hline
\end{tabular}


Anexo 3: Población

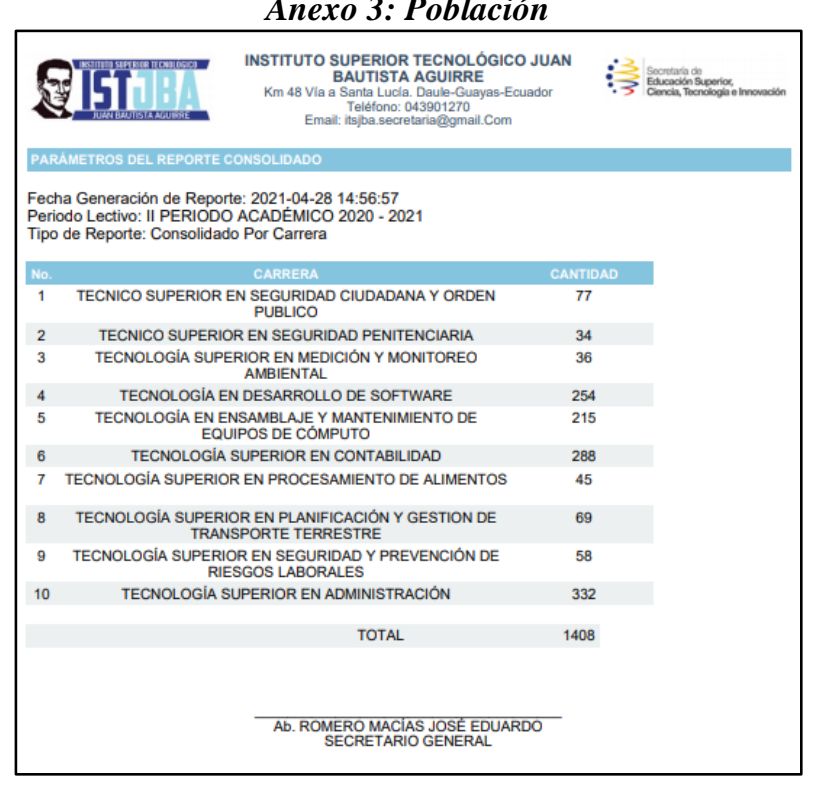

Anexo 3: Google formularios - Encuesta

Link:

https://docs.google.com/forms/d/e/1FAIpQLScWY29YmUFwBmIGdHtZMxaVy7RwHrEU Mc1hvmsMWIEZVJ2ihw/viewform?usp=sf_link 